\title{
Lactobacillus casei DG and its postbiotic reduce the inflammatory mucosal response: an ex-vivo organ culture model of post- infectious irritable bowel syndrome
}

\author{
Debora Compare, Alba Rocco, Pietro Coccoli, Debora Angrisani, Costantino Sgamato, Barbara lovine,
} Umberto Salvatore and Gerardo Nardone*

\begin{abstract}
Background: The evidence on the role of gut microbiota in post-infectious irritable bowel syndrome (PI-IBS) is convincing. Lactobacillus spp. positively affect IBS symptoms, although the mechanisms through which probiotics exert their beneficial effects are largely unknown. The aim of the study is to evaluate the role of Lactobacillus casei DG (LC-DG) and its postbiotic (PB) in modulating the inflammatory/immune-response in PI-IBS in an ex-vivo organ culture model.

Methods: Ex vivo cultures of ileal and colonic mucosa from 10 PI-IBS, diarrhea predominant subtype (D) patients, and 10 healthy controls (HC) were treated with LPS, LC-DG and PB. Interleukin (IL)-1a, IL-6, IL-8 and IL-10 mRNA levels were assessed by real-time PCR and Toll like receptor 4 (TLR-4) protein expression by Western blotting.

Results: At baseline, IL-1a, IL-6 and IL-8 mRNA levels as well as TLR-4 protein expression were significantly higher while IL-10 mRNA levels were lower in PI-IBS D than in HC in both ileum and colon. LC-DG and PB significantly reduced the mRNA levels of pro-inflammatory cytokines and TLR-4 while increased that of IL-10 after LPS stimulation. The protective effect was more pronounced for PB than LC-DG treatment.
\end{abstract}

Conclusion: LC-DG and its PB attenuate the inflammatory mucosal response in an ex-vivo organ culture model of PI-IBS D.

Keywords: Post-infectious irritable bowel syndrome, Probiotics, Postbiotic

\section{Background}

Irritable bowel syndrome (IBS) is the commonest functional gastrointestinal disorder, affecting up to $20 \%$ of the population in Europe and the USA [1]. IBS does not predispose patients to severe illness but it deeply affects the quality of life and incurs a significant economic burden in both direct and indirect expenditures worldwide [2]. According to the symptom's pattern IBS is subcategorized into IBS with constipation (IBS-C), IBS with diarrhea (IBS-D), mixed IBS (IBS-M) and unsubtyped IBS (IBS-U) [3]. Gastrointestinal dysmotility, visceral hypersensitivity, brain-gut axis dysfunction, and

\footnotetext{
* Correspondence: nardone@unina.it

Department of Clinical Medicine and Surgery, Gastroenterology Unit,

University Federico II of Naples, Via S. Pansini 5, 80131 Naples, Italy
}

alterations in psychosocial or psychosomatic behavior have been implicated in the pathophysiology of IBS, but the exact mechanisms remain largely undefined [4]. Recently, IBS research focused on causative factors such as low-grade mucosal inflammation and local immune activation [5], both triggered by perturbations of gut microbiota. The evidence for gut microbes playing a role in the pathogenesis of IBS is convincing. Small intestinal bacterial overgrowth (SIBO) is frequently detected in IBS patients and symptoms pattern of SIBO largely overlap those of IBS [6]. Recently, qualitative alterations of gut microbiota in subjects with IBS were also revealed by metagenomic approaches [7]. Based on the assumption that IBS develops in up to $30 \%$ of individuals recovering from acute gastroenteritis, the pathogenetic role of gut 
microbiota was well established mainly for post-infectious (PI)-IBS, diarrhea (D) predominant subtype [8]. As a consequence, the manipulation of the microbiota is becoming an attractive therapeutic option for this disease [9]. A recent systematic review focusing on the efficacy of Lactobacillus spp in IBS found a positive effect on symptom relief [10]. However, few studies have addressed the mechanisms through which probiotics exert their beneficial effects. Moreover, growing data, mainly obtained by the analysis of Lactobacilli strains, support the evidence that these beneficial effects may depend on secreted probiotic-derived factors, recently identified as postbiotic (PB) mediators [11].

\section{Methods}

Aim of this study was to evaluate the role of Lactobacillus casei DG (LC-DG) and its PB in modulating the inflammatory immune-response in an ex-vivo organ culture model of PI-IBS D. Patients with a diagnosis of PI-IBS who met Rome III criteria for IBS-D were consecutively recruited among 92 patients with IBS referred to the Gastroenterology Unit of the University Federico II of Naples from December 2014 to June 2015. A total of 10 out of 13 PI-IBS patients (6 males; mean age 52 years) accepted to participate to the study. The patients confirmed IBS onset after an episode of acute gastroenteritis with diarrhea and/or vomiting occurred at least one year before the enrollment, thus fulfilling the definition of PI-IBS [12]. Ten control subjects (5 males; mean age 48 years) were recruited among those referred to perform a colonoscopy indicated within the framework of colorectal cancer screening (age, anemia, positivity of the fecal occult blood test, rectal bleeding, personal or family history of polyps, family history of colorectal cancer). Inclusion criteria were age $\geq 18$ and $\leq 70$ years and normal findings at endoscopic and histological examinations. Presence of any of the following criteria excluded patients from the study: ascertained inflammatory bowel diseases (Crohn's disease, diverticular disease, ulcerative colitis, ischemic colitis, microscopic colitis, coeliac disease), topic or systemic antibiotic and probiotic therapy during the last month, therapy with nonsteroidal anti-inflammatory drugs, proton pump inhibitors or H2-antagonists, antiplatelet and/or anticoagulant drugs, intended or ascertained pregnancy or lactation, copro-parasitological examination of stools positive, active malignancy of any kind, or a history of a malignancy, clinically relevant renal, hepatic, haematologic, cardiac, neurological, psychiatric, immunological, gastrointestinal, metabolic or endocrine disease, abuse of alcohol, drugs or psychotropic drugs which may affect alertness and physical perception and inability to conform to the protocol or denied consensus. All subjects underwent ileo-colonoscopy with multiple bioptic sampling at ileal and left colon sites for both histology and ex vivo organ culture.

\section{Probiotic and postbiotic preparation}

Lactobacillus Casei DG was obtained dried by SOFAR (Milan, Italy) and stored at a temperature below $25^{\circ} \mathrm{C}$ until use. Bacteria were restarted at 1:100 and grown in MRS broth (Biokar Diagnostic, Beauvais Cedex, France) to an $\mathrm{OD} 600=0.6$. Bacterial cultures were plated to count effective colony forming units (CFUs). The growth curve was evaluated after $24 \mathrm{~h}$ at $37{ }^{\circ} \mathrm{C}$ in conventional bacterial incubation or in a $5 \% \mathrm{CO} 2$ incubator for eukaryotic cells or in oxygen chamber, which was filled with pressurized oxygen. The growth profile was determined by the calculation of the specific growth rate $(u)$ that is the change in the number of cells in unit of time $\mathrm{u}=(\mathrm{OD} 1 / \mathrm{OD} 2) /(\mathrm{T} 2-\mathrm{T} 1)$. Postbiotic was obtained by centrifugation at $10,000 \mathrm{xg}$ for $15^{\prime}$ of a LC-DG culture in exponential phase with equivalent amount of CFUs.

\section{Experimental procedures}

The experimental procedures are summarized in Fig. 1. Mucosal bioptic samples from ileum and left colon were immediately placed on culture filter plates $(15 \mathrm{~mm}$ diameter wells with $500 \mathrm{~mm}$ bottom-mesh, Netwell culture system, Costar, Cambridge, MA, USA) with the epithelial surface uppermost. Filters were placed into wells containing $1 \mathrm{ml}$ RPMI (Gibco Laboratories, North Andover, MA), 10\% fetal bovine serum (FBS) (Gibco Laboratories, North Andover, MA, USA) in presence or absence of $100 \mu \mathrm{g} / \mathrm{ml}$ lipopolysaccharides (LPS) (SigmaAldrich, Milan, Italy), and without antibiotics for $2 \mathrm{~h}$ at $37{ }^{\circ} \mathrm{C}$ in a $5 \%$ carbon dioxide incubator. Then, the medium was substituted with RPMI with $10 \%$ FBS, containing LC-DG (1x107 CFU) or the PB or equal volume of unfermented bacterial medium MRS medium as control. After $2 \mathrm{~h}$ at $37{ }^{\circ} \mathrm{C}$ in a $5 \% \mathrm{CO} 2$ incubator, the medium was removed and replaced with RPMI 10\% FBS, containing 3\% penicillin/streptomycin (Gibco Laboratories, North Andover, MA, USA) and $50 \mu \mathrm{g} / \mathrm{ml}$ gentamycin. The tissues were then transferred into the oxygen chamber, which was filled with pressurized oxygen (VitalAire, Milan, Italy) and placed at $37{ }^{\circ} \mathrm{C}$ for the remaining $19 \mathrm{~h}$ of culture. The control samples were treated in the same way without addition of LPS, LC-DG or PB.

\section{RNA and protein extracts preparation}

Total RNA and protein extracts were prepared from intestinal biopsies by using the TRIzol Reagent (Life Technology, Carlsbad, CA, USA). Briefly, each biopsy was placed in $200 \mu \mathrm{l}$ of TRIzol Reagent and homogenized using a glass Teflon. Following homogenization the samples were centrifuged at $12,000 \mathrm{~g}$ for $10^{\prime}$ at $4{ }^{\circ} \mathrm{C}$ and the 


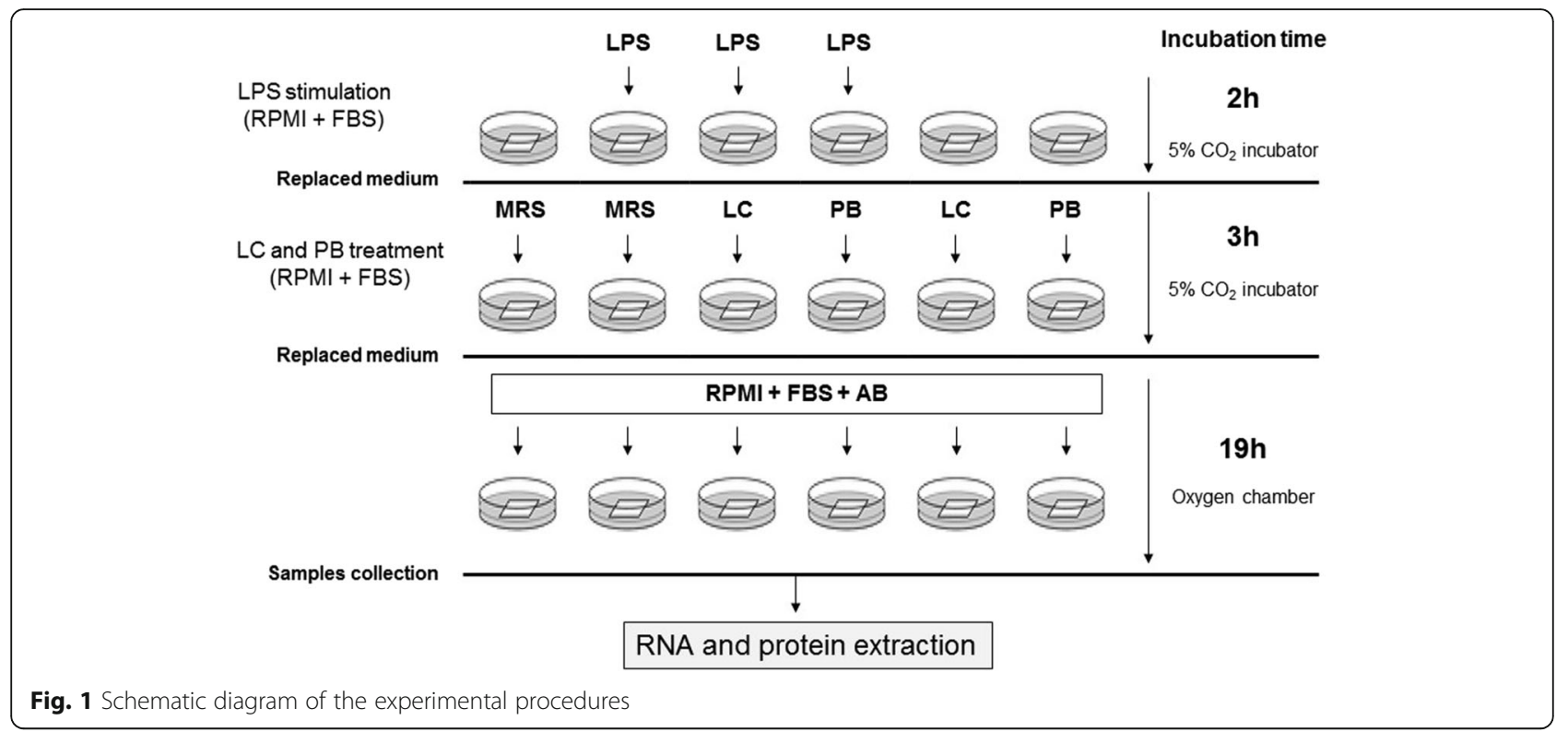

aqueous phase, containing RNA, and the organic interphase, containing proteins, were processed separately according to the data sheet protocol. Total RNA quantity and quality were evaluated by RNA nanodrop and displaying on denaturing agarose gel. RNAs were stored at $-80{ }^{\circ} \mathrm{C}$ until use for Real Time.

\section{Real-time PCR}

$1 \mu \mathrm{g}$ total RNA was used to synthesize the cDNA according to the iScript cDNA Synthesis kit protocol (Bio-Rad Laboratories, Hercules, CA, USA). The cDNA was then amplified in an iCycler iQ real time PCR detection system (Bio-Rad Laboratories, Hercules, CA, USA) by using iQTM SYBR Green Supermix (Bio-Rad Laboratories, Hercules, CA, USA). The Real-Time PCR reactions and the relative quantification gene expression were performed as previously reported [13]. Each experiment was performed in triplicate. Relative quantification of gene expression was performed using the 2- $\Delta \Delta C T$ method. The primer sequences of the analyzed interleukins (ILs) are reported in Table 1.

\section{Western blotting analysis}

Protein concentrations were determined by a Bio-Rad protein assay (Bio-Rad Laboratories, Hercules, CA,

Table 1 Primers used for real time PCR experiments

\begin{tabular}{ll}
\hline IL-1a & F:5'-CGCCAATGACTCAGAGGAAGA-3' \\
& R:5'-AGGGCGTCATTCAGGATGAA-3' \\
IL-6 & F:5'-TACCCCCAGGAGAAGATCC-3' \\
& R:'-GCCATCTTTGGAAGGTCAG-3' \\
IL-8 F:5'-AGACAGCAGAGCACACAAGC & R:5'-ATGGTTCCCCTTCCGGTGGT-3' \\
& F:5'-GAACCAAGACCCAGACATC-3' \\
IL-10 R:5'-CATTCTTCTCACCTGCTCCAC-3' & \\
\end{tabular}

USA) and $20 \mu \mathrm{g}$ were diluted with loading buffer and heated to $95{ }^{\circ} \mathrm{C}$ for $10^{\prime}$. Then, protein lysates were run onto 10\% SDS-PAGE gel and transferred on PVDF membrane (MILLIPORE). Membranes were probed overnight at $4{ }^{\circ} \mathrm{C}$ with rabbit anti-TLR4 (1:1,000; Novus, Littleton, CO, USA) and rabbit anti- $\alpha$-actin $(1: 1,000$; Santa Cruz Biotechnology, Dallas, TX) antibodies. Each experiment was performed in triplicate. The signals were detected after the incubation with antirabbit peroxidase-conjugated secondary antibodies (1:5,000; Santa Cruz Biotechnology, Dallas, TX, USA) by using the ECL kit (Advansta, Menlo Park, CA, USA). The band densities were assessed using Image J 1.40 g software.

\section{Statistical analysis}

All data collected were summarized separately for each patient in all experimental conditions and expressed as mean \pm standard deviation. Mann-Whitney $U$ test and one-way ANOVA, when appropriate, were used to compare the variables. A $p$ value $<0.05$ was set as level of significance. Statistical analysis was performed with SPSS for Windows (version 19.0; IBM Corporation, Armonk, NY, USA). Prism for Windows 5 (version 5.02; GraphPad Software Inc., La Jolla, CA, USA) was used for graphical presentation.

\section{Results}

\section{Effect of LC-DG and PB on IL-1a, IL-6, IL-8 mRNA and} IL-10 mRNA levels

At baseline, IL-1 $\alpha$, IL- 6 and IL- 8 mRNA levels were higher while IL-10 mRNA levels were lower in PI-IBS D than $\mathrm{HC}$, irrespective of intestinal mucosa site (Figs. 2 and 3). Notably, in PI-IBS D patients, IL-6 mRNA levels 

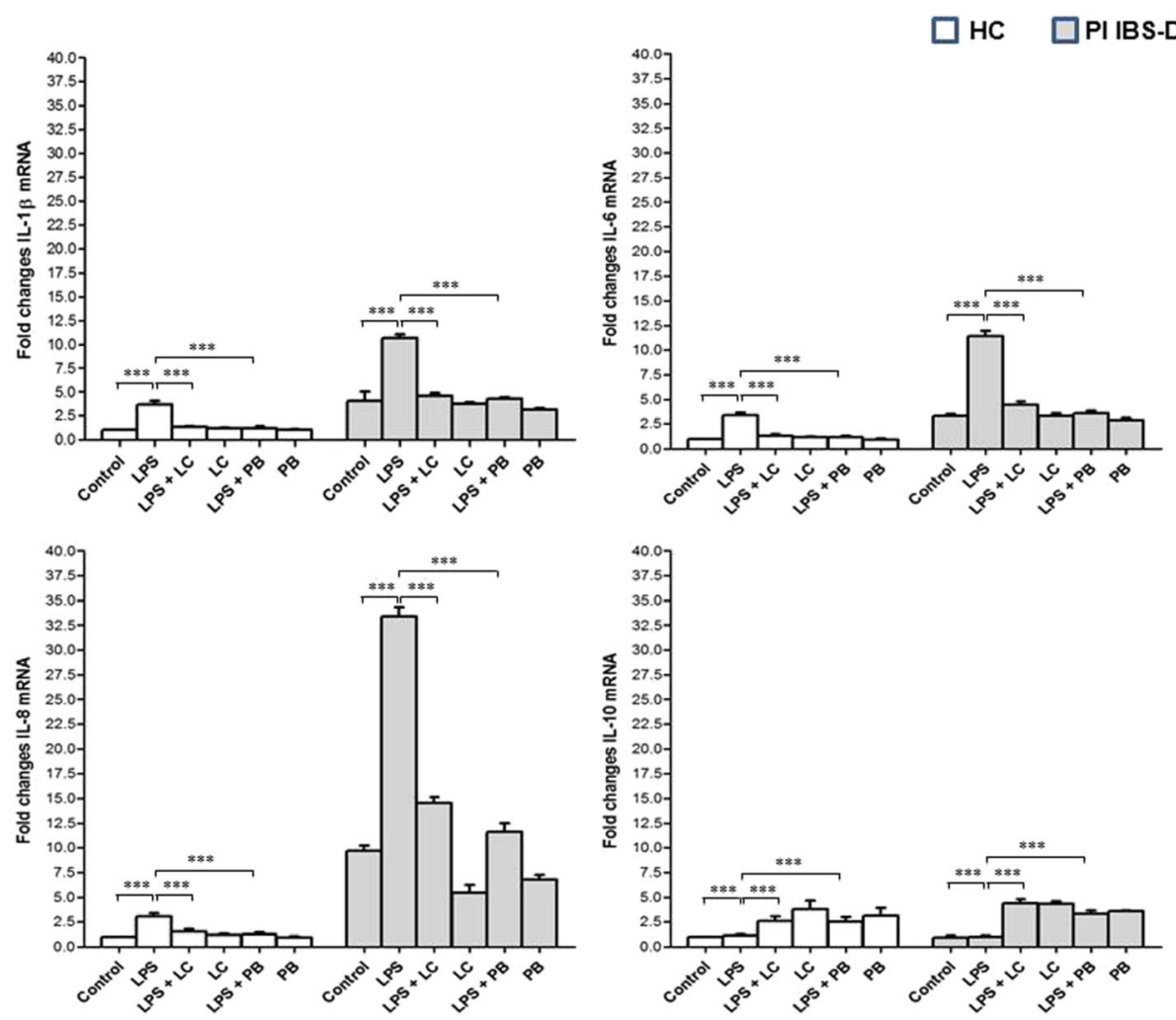

Fig. 2 Fold changes in mRNA levels of IL-1a, IL-6, IL-8 and IL-10 in the ileal mucosa of HC and IBS-D patients. IL-1a, IL-6 and IL-8 mRNA baseline levels were higher and IL-10 mRNA levels were lower in post-infectious IBS-D than HC. The stimulation of intestinal mucosa with $100 \mu \mathrm{g} / \mathrm{ml}$ LPS significantly increased mRNA levels of all cytokines in respect to baseline in both HC and PI-IBS D patients. In contrast, LPS treatment did not affect IL-10 mRNA levels in both HC and IBS-D. LC-DG treatment was effective in reducing IL-1a and IL-8 mRNA levels and increasing IL-10 m-RNA levels. PB treatment was effective in reducing IL-1a, IL-6 and IL-8 mRNA levels and increasing IL-10 m-RNA levels. *** $p<0.0001$. HC: healthy controls; PI IBS-D: post-infectious irritable bowel disease diarrhea subtype; LPS: lipopolysaccharide; LC: Lactobacillus Casei DG; PB: postbiotic

were higher in colonic than in ileal mucosa while IL-8 mRNA levels were higher in ileal than in colonic mucosa. The stimulation of intestinal mucosa with $100 \mu \mathrm{g} / \mathrm{ml}$ LPS significantly increased mRNA levels of all cytokines in respect to baseline in both $\mathrm{HC}$ and PI-IBS D patients (Figs. 2 and 3). However, the magnitude of the inflammatory response of the intestinal mucosa, that is the difference between LPS-induced mRNA levels and baseline values, was greater in patients than in $\mathrm{HC}$ in both ileal and colonic mucosa (Il- $1 \alpha$ $p<0.0001$, IL-6 $p<0.0001$ and IL-8 $p<0.0001$ ). In contrast, the magnitude of the anti-inflammatory response did not significantly differ between HC and IBS-D, irrespective of mucosal site.

In PI-IBS D, the treatment of colonic biopsies with LC-DG significantly reduced the levels of all proinflammatory cytokines (Il-1 $\alpha p<0.002$, IL-6 $p<0.0001$ and IL-8 $p<0.0001)$ in respect to baseline. In ileal mucosa, LC-DG treatment was effective in reducing IL$1 \alpha$ and IL- 8 mRNA levels $(p<0.0002$ and $p<0.0001$, respectively) but did not affect IL-6 mRNA levels. LC-DG treatment significantly increased IL-10 m-RNA levels in both colonic and ileal mucosa $(p<0.0001$ and $p<0.0001$, respectively). Similarly, PB treatment was effective in reducing IL- $1 \alpha$, IL- 6 and IL- 8 mRNA levels in both colonic $(p<0.0001, p<0.0001$ and $p<0.0001$, respectively) and ileal mucosa $(p<0.0001, p<0.0006$ and $p<0.0001$, respectively). In contrast, IL-10 m-RNA levels significantly increased in both ileal and colonic mucosa $(p<0.0001$ and $p<0.0001$, respectively). The protective effect of LC-DG and PB was not affected by the pre-treatment of intestinal biopsies with LPS. Interestingly, the effect was more pronounced for $\mathrm{PB}$ treatment in respect to LC-DG treatment, in all cases.

\section{Effect of LC-DG and PB on TLR-4 protein expression after LPS stimulation}

At baseline, TLR-4 protein expression was significantly higher in PI-IBS D patients in respect to $\mathrm{HC}$ in both ileal and colonic mucosa. In details, TLR-4 protein expression was 7.4-folds higher in ileal mucosa $(p<0.0001)$ and 3 -folds higher in colonic mucosa $(p<0.001)$ of PI-IBS D patients as compared with HC. LPS stimulation significantly increased TLR-4 protein expression in both HC 

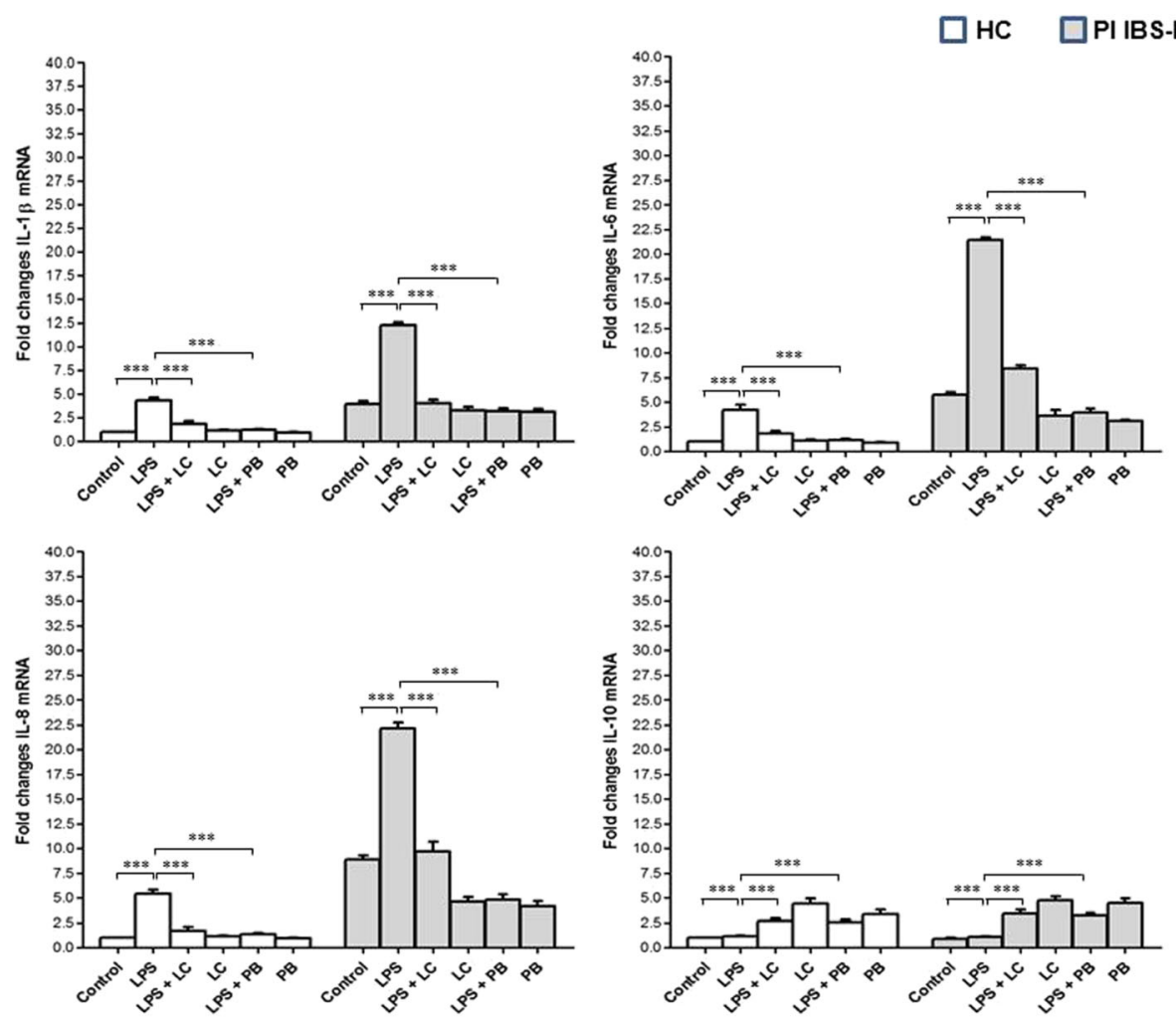

Fig. 3 Fold changes in mRNA levels of IL-1a, IL-6, IL-8 and IL-10 in the left colon mucosa of HC and IBS-D patients. IL-1a, IL-6 and IL-8 mRNA baseline levels were higher and IL-10 mRNA levels were lower in PI-IBS D than HC. The stimulation of intestinal mucosa with $100 \mu \mathrm{g} / \mathrm{ml}$ LPS significantly increased mRNA levels of all cytokines in respect to baseline in both HC and PI-IBS D patients. In contrast, LPS treatment did not affect IL-10 mRNA levels in both HC and IBS-D. LC-DG and PB treatment were effective in reducing IL-1a, IL-6 and IL-8 mRNA levels and increasing IL-10 m-RNA levels. ${ }^{* * *} p<0.0001$. HC: healthy controls; PI IBS-D: post-infectious irritable bowel disease diarrhea subtype; LPS: lipopolysaccharide; LC: Lactobacillus Casei DG; PB: postbiotic

and IBS-D patients $(p<0.0001)$ with a more pronounced effect in colonic mucosa. The increase of TLR-4 protein expression was attenuated by LC-DG and PB treatment in both $\mathrm{HC}$ and IBS-D patients $(p<0.0001)$. Interestingly, the protective effect was more evident in ileal than in colonic mucosa (Fig. 4).

\section{Discussion}

In our study, the baseline expression of pro-inflammatory cytokines, namely IL- $1 \alpha$, Il- 6 and Il- 8 was significantly higher and that of IL-10 significantly lower in the intestinal mucosa of PI-IBS D patients in respect to HC.

An increased number of lamina propria lymphocytes and mRNA levels of pro-inflammatory cytokines with a parallel decrease of mRNA levels of anti-inflammatory cytokines have been described in colonic mucosa of PI-IBS as compared to non-PI-IBS and control subjects [14, 15].

Moreover, PI-IBS patients had an increased expression of IL- $1 \alpha$ mRNA in terminal ileum and recto-sigmoidal mucosa [16]. These data support the hypothesis that the immune dysfunction and the dysregulated neuroimmune interactions may predispose individuals to IBS [8]. Based on this pathophysiological model an event, such as infection, would generate permanent disturbances of the gut microbiota with overgrowth of pathogens and a marked reduction in bacterial diversity. The breakdown of the microbial ecology might be responsible for the "chronic low grade inflammation" of the intestinal mucosa, typically observed in IBS patients. In addition, an altered host-microbiota interaction, may also contribute to the pathophysiology of IBS.

TLRs represent a first line of host defense to pathogens by activating responses in cells of the innate immune system [17]. Brint et al. firstly reported a 4-fold increase in the TLR-4 mRNA expression in rectosigmoid mucosa of IBS patients as compared with controls [18]. However, this study did not analyze the right colon and terminal ileum mucosa, the site of highest concentrations of bacteria and where most immunological events occur. Moreover, even if PI-IBS could represent the most relevant subtype for the assessment of TLR expression, due to the infectious origin, this subgroup of patients was excluded from the study. In our study, TLR-4 protein expression was significantly higher in PI-IBS D patients in respect to $\mathrm{HC}$, in both ileal and colonic mucosa. Interestingly, while in the $\mathrm{HC}$ group the TLR-4 protein expression 


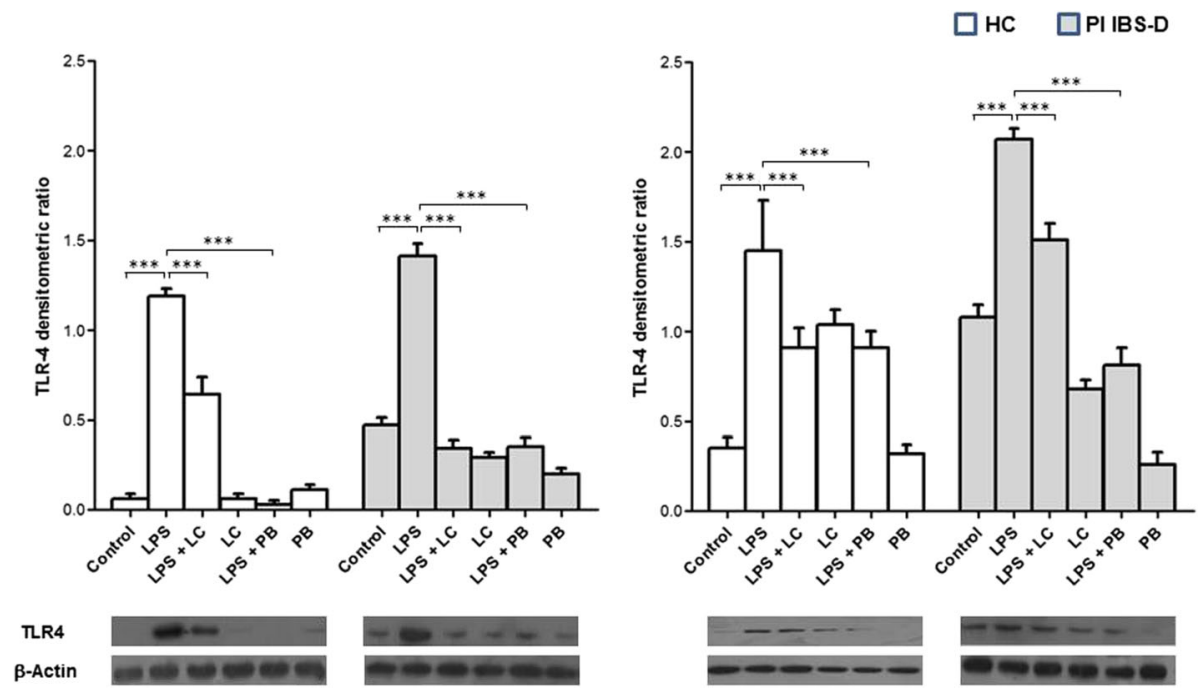

Fig. 4 TLR-4 protein expression in ileal and left colon mucosa of HC and IBS-D patients. TLR-4 protein baseline levels were higher in PI-IBS D patients in respect to HC in both ileal and colonic mucosa. The stimulation of intestinal mucosa with $100 \mu \mathrm{g} / \mathrm{ml}$ LPS increased TLR-4 protein expression in both HC and IBS-D patients with a more pronounced effect in colonic mucosa. LC-DG and PB treatment reduced TLR-4 protein expression in both HC and IBS-D patients particularly in ileal mucosa. ${ }^{* *} p<0.0001$. HC: healthy controls; PI IBS-D: post-infectious irritable bowel disease diarrhea subtype; LPS: lipopolysaccharide; LC: Lactobacillus Casei DG; PB: postbiotic

did not significantly differ between ileal and colonic mucosa, in PI-IBS D patients we found a significantly increased TLR-4 protein expression in the ileal mucosa.

Our data are in line with both basic and clinical evidence on the causative role of intestinal dysbiosis in the pathogenesis of IBS, thus supporting the idea that the modulation of gut microbiota could be an attractive treatment option for the disease. Randomised controlled trials demonstrated that probiotics were effective in the modulation of symptom's pattern of IBS patients [19-24]. However, the majority of the studies have been performed in non-specific IBS rather than in PI-IBS D, so that the exact role of probiotics in the management of these patients remains to be elucidated. In addition, the subjective nature of the symptoms used as surrogate end-points in clinical trials does not prove the "anti-inflammatory" effect of probiotics in IBS. On the other hand, the assessment of the effect of probiotics on intestinal mucosa would require repeated endoscopic examinations with extensive bioptic sampling, that is unfeasible because of patient acceptability and ethical concerns. Probiotics action has been studied on isolated cells and cell lines, cell co-cultures and mouse models [25-27]. These models do not accurately represent the unique microenvironment of the intestine as they all lack important human-specific components like the mucus and the microbiota [27]. The development of a model system that resembles the human intestine is therefore of great value for testing the action of probiotics on both healthy and diseased tissues. The use of entire highly viable human intestinal biopsy specimens in culture is the ideal model, especially to analyze biological phenomena occurring within the first 24-48 h after which the epithelium in the biopsy specimens is no more viable [11]. By using such a model we analysed the effect of the probiotic strain LC-DG on ileal and colonic mucosa of PI-IBS D patients. The strain we used in the experimental setting was selected on the basis of its human origin, non-pathogenicity, resistance to intestinal acid and bile, ability to adhere to human epithelial cells and colonize the human gastrointestinal tract. Several studies found a decrease of Lactobacilli spp. in faeces and mucosa of IBS patients [28-30], while, interventional trials demonstrated the capability of Lactobacilli to improve symptoms and modulate the inflammatory response in these patients [10]. However, to the best of our knowledge, no data are available on the effect of LC-DG on the mucosal inflammatory response. In our study, we demonstrated that treatment of intestinal biopsies with LC-DG significantly reduced mRNA levels of all proinflammatory cytokines as well as the TLR-4 protein expression while increased the mRNA levels of the anti-inflammatory IL-10 in both ileal and colonic mucosa of PI-IBS D patients. Interestingly, the pretreatment of intestinal biopsies with LPS did not affect the anti-inflammatory action of LC-DG.

Probiotics act through molecular and cellular mechanisms that contrast pathogen bacteria adhesion,

enhance innate immunity, decrease pathogen-induced inflammation, and promote intestinal epithelial cell survival, barrier function, and protective responses. However, Lactobacilli-derived PB mediators seem to 
mediate the beneficial effects of probiotics [11]. Interestingly, in our study, the modulatory effect on inflammatory response of the intestinal mucosa was even more successful when we used the PB obtained from LC-DG cultures.

We are aware that the relatively small number of patients included in our study prevents us to generalize our results. However, strengths of our study are the rigorous selection of the patients, the "ex vivo" organ culture model we used, the sampling of both ileal and left colon mucosa and the analysis of the effect of both the probiotic strain LC-DG and its PB on the inflammatory response of the intestinal mucosa.

\section{Conclusions}

LC-DG and its PB attenuate the inflammatory mucosal response in an ex-vivo organ culture model of PI-IBS D. These findings provide biological plausibility to the therapeutically usefulness of this probiotic strain in the clinical setting of PI-IBS D. Well designed and powered clinical trials are advisable to confirm the therapeutic efficacy in the real life management of these patients.

\begin{abstract}
Abbreviations
FBS: Fetal bovine serum; HC: Healthy controls; IBS: Irritable bowel syndrome; IBS-C: Irritable bowel syndrome IBS with constipation; IBS-D: Irritable bowel syndrome IBS with diarrhea; IBS-M: Mixed irritable bowel syndrome; IBSU: Unsubtyped irritable bowel syndrome; L: Interleukin; LC-DG: Lactobacillus casei DG; LPS: Lipopolysaccharides; LPS: Postbiotic; PI-IBS D: Post infectious irritable bowel syndrome diarrhea predominant subtype; PIIBS: Postinfectious irritable bowel syndrome Irritable bowel syndrome; SIBO: Small intestinal bacterial overgrowth; TLRs: Toll like receptors
\end{abstract}

\section{Acknowledgments}

Not applicable.

\section{Funding}

SOFAR S.p.A.

\section{Availability of data and materials}

The dataset generated during the present study is available upon reasonable request to the corresponding author (Prof. G. Nardone).

\section{Authors' contributions}

DC Study concept and design, analysis and interpretation of data, drafting of the manuscript, Statistical analysis. AR Study concept and design, analysis and interpretation of data, drafting of the manuscript, Statistical analysis. PC Patient recruitment, data collection and drafting of the manuscript. DA Patient recruitment, data collection and drafting of the manuscript. CS Patient recruitment, data collection and drafting of the manuscript. BI Experimental procedures and analysis and interpretation of data. US Experimental procedures and analysis and interpretation of data. GN Critical revision of the manuscript, drafting of the manuscript, study supervision. All authors have read and approved the final version of this manuscript.

\section{Competing interests}

GN received funding by SOFAR S.p.A. to support the experimental procedures of the study.

\section{Consent for publication}

Not applicable.

\section{Ethics approval and consent to participate}

The study protocol was approved by the Ethical Committee "Carlo Romano" of University Federico II, Naples (protocol n. 150/13). Written informed consent was obtained from all partecipants.

\section{Publisher's Note}

Springer Nature remains neutral with regard to jurisdictional claims in published maps and institutional affiliations.

Received: 9 November 2016 Accepted: 28 March 2017

Published online: 14 April 2017

\section{References}

1. Lovell RM, Ford AC. Global prevalence of and risk factors for irritable bowel syndrome: a meta-analysis. Clin Gastroenterol Hepatol. 2012;10:712-721.e4.

2. Canavan C, West J, Card T. Review article: the economic impact of the irritable bowel syndrome. Aliment Pharmacol Ther. 2014;40:1023-34.

3. Shih DQ, Kwan LY. All roads lead to Rome: update on Rome III criteria and new treatment options. Gastroenterol Rep. 2007;1:56-65.

4. Chey WD, Kurlander J, Eswaran S. Irritable bowel syndrome: a clinical review. JAMA. 2015;313:949-58.

5. Dupont HL. Review article: evidence for the role of gut microbiota in irritable bowel syndrome and its potential influence on therapeutic targets. Aliment Pharmacol Ther. 2014;39:1033-42.

6. Ford AC, Spiegel BM, Talley NJ, Moayyedi P. Small intestinal bacterial overgrowth in irritable bowel syndrome: systematic review and metaanalysis. Clin Gastroenterol Hepatol. 2009;7:1279-86.

7. Collins SM. A role for the gut microbiota in IBS. Nat Rev Gastroenterol Hepatol. 2014;11:497-505.

8. Spiller R, Garsed K. Postinfectious irritable bowel syndrome. Gastroenterology. 2009;136:1979-88.

9. Distrutti E, Monaldi L, Ricci P, Fiorucci S. Gut microbiota role in irritable bowel syndrome:New therapeutic strategies. World J Gastroenterol. 2016;22:2219-41.

10. Tiequn B, Guanqun C, Shuo Z. Therapeutic effects of Lactobacillus in treating irritable bowel syndrome: a meta-analysis. Intern Med. 2015;54:243-9.

11. Tsilingiri K, Barbosa T, Penna G, et al. Probiotic and postbiotic activity in health and disease: comparison on a novel polarised ex-vivo organ culture model. Gut. 2012;61:1007-15.

12. Spiller RC. Inflammation as a basis for functional GI disorders. Best Pract Res Clin Gastroenterol. 2004;18:641-61.

13. lovine $B$, Nino $M$, Irace $C$, et al. Ultraviolet $B$ and $A$ irradiation induces fibromodulin expression in human fibroblasts in vitro. Biochimie. 2009;91:364-72.

14. Sundin J, Rangel I, Fuentes S, et al. Altered faecal and mucosal microbia composition in post-infectious irritable bowel syndrome patients correlates with mucosal lymphocyte phenotypes and psychological distress. Aliment Pharmacol Ther. 2015;41:342-51.

15. Sundin J, Rangel I, Repsilber D, Brummer RJ. Cytokine response after stimulation with key commensal bacteria differ in post-Infectious Irritable Bowel Syndrome (PIIBS) patients compared to healthy controls. PLoS One. 2015;10:e0134836.

16. Gwee KA, Collins SM, Read NW, et al. Increased rectal mucosal expression of interleukin 1 beta in recently acquired post-infectious irritable bowel syndrome. Gut. 2003;52:523-6.

17. Kawai T, Akira S. The role of pattern-recognition receptors in innate immunity: update on toll-like receptors. Nat Immunol. 2010;11:373-84.

18. Brint EK, MacSharry J, Fanning A, et al. Differential expression of toll-like receptors in patients with irritable bowel syndrome. Am J Gastroenterol. 2011:106:329-36.

19. Stevenson C, Blaauw R, Fredericks E, et al. Randomized clinical trial: effect of Lactobacillus plantarum $299 \mathrm{v}$ on symptoms of irritable bowel syndrome. Nutrition. 2014;30:1151-7.

20. Sisson G, Ayis S, Sherwood RA, Bjarnason I. Randomised clinical trial: a liquid multi-strain probiotic vs. placebo in the irritable bowel syndrome-a 12 week double-blind study. Aliment Pharmacol Ther. 2014;40:51-62.

21. Ludidi S, Jonkers DM, Koning CJ, et al. Randomized clinical trial on the effect of a multispecies probiotic on visceroperception in hypersensitive IBS patients. Neurogastroenterol Motil. 2014;26:705-14.

22. Begtrup LM, de Muckadell OB, Kjeldsen J, et al. Long-term treatment with probiotics in primary care patients with irritable bowel syndrome- a randomised, double-blind, placebo controlled trial. Scand J Gastroenterol. 2013:48:1127-35.

23. Yoon JS, Sohn W, Lee OY, et al. Effect of multispecies probiotics on irritable bowel syndrome: a randomized, double-blind, placebo-controlled trial. J Gastroenterol Hepatol. 2014;29:52-9. 
24. Fong FL, Shah NP, Kirjavainen P, El-Nezami H. Mechanism of action of probiotic bacteria on intestinal and systemic immunities and antigenpresenting cells. Int Rev Immunol. 2015;25:1-11.

25. Giorgetti G, Brandimarte $G$, Fabiocchi F, et al. Interactions between innate immunity, microbiota, and probiotics. J Immunol Res. 2015;2015:501361.

26. Frei R, Akdis M, O'Mahony L. Prebiotics, probiotics, synbiotics, and the immune system: experimental data and clinical evidence. Curr Opin Gastroenterol. 2015;31:153-8.

27. Tsilingiri K, Rescigno M. Should probiotics be tested on ex vivo organ culture models? Gut Microbes. 2012;3:442-8.

28. Shukla R, Ghoshal U, Dhole TN, Ghoshal UC. Fecal microbiota in patients with irritable bowel syndrome compared with healthy controls using realtime polymerase chain reaction: an evidence of dysbiosis. Dig Dis Sci. 2015;60:2953-62.

29. Jeffery IB, O'Toole PW, Öhman L, et al. An irritable bowel syndrome subtype defined by species-specific alterations in faecal microbiota. Gut. 2012;61:997-1006.

30. Tana C, Umesaki Y, Imaoka A, et al. Altered profiles of intestinal microbiota and organic acids may be the origin of symptoms in irritable bowel syndrome. Neurogastroenterol Motil. 2010;22:512-9.

Submit your next manuscript to BioMed Central and we will help you at every step:

- We accept pre-submission inquiries

- Our selector tool helps you to find the most relevant journal

- We provide round the clock customer support

- Convenient online submission

- Thorough peer review

- Inclusion in PubMed and all major indexing services

- Maximum visibility for your research

Submit your manuscript at www.biomedcentral.com/submit
() BioMed Central 